Citation: Ahmad FS. (2018) "Reversal of fixed pulmonary hypertension with transcatheter valve replacement for aortic insufficiency on ventricular assist device support"

The VAD Journal, 4. doi: https://doi.org/10.13023/VAD.201 8.05

Editor-in-Chief: Maya Guglin, University of Kentucky

Received: April 2, 2018

Accepted: June 12, 2018

Published: June 17, 2018

(c) 2018 The Author(s). This is an open access article published under the terms of the Creative Commons Attribution-

NonCommercial 4.0 International License

(https://creativecommons.org/lice nses/by-nc/4.0/), which permits unrestricted non-commercial use, distribution, and reproduction in any medium, provided that the original author(s) and the publication source are credited.

Funding: Not applicable

Competing interests: Not applicable
Peer-Reviewed Case report

\section{Reversal of fixed pulmonary hypertension with transcatheter valve replacement for aortic insufficiency on ventricular assist device support}

Faraz S. Ahmad ${ }^{1 *}$, Mark J. Ricciardi ${ }^{1}$, Laura J. Davidson ${ }^{1}$, Allen S. Anderson 1, Kambiz Ghafourian', Ike Okwuosa1, Esther Vorovich', Jane E. Wilcox ${ }^{1}$, Daniel D. Holloway ${ }^{2}$, Duc T. Pham ${ }^{2}$, and Jonathan D. Rich ${ }^{1}$

*Corresponding author: faraz.ahmad@northwestern.edu

${ }^{1}$ Department of Medicine, Division of Cardiology, Northwestern University Feinberg School of Medicine, Chicago, Illinois, USA

${ }^{2}$ Department of Surgery, Division of Cardiothoracic Surgery, Northwestern University, Chicago, Illinois, USA

\begin{abstract}
We present a 43-year-old woman with a nonischemic cardiomyopathy implanted with a ventricular assist device (VAD) as bridge to transplant due to severe, "fixed" pulmonary hypertension $(\mathrm{PH})$. Within three months of VAD implant, her "fixed" PH had resolved entirely. Nearly two years later, still supported with a VAD because of severe HLA allosensitization, she developed dyspnea and "moderate" aortic insufficiency (AI) by standard criteria. Invasive hemodynamics revealed recurrence of severe $\mathrm{PH}$ in the setting of elevated left-sided filling pressures. We concluded the Al was indeed severe and the cause of her symptoms and recurrent $\mathrm{PH}$. Despite her noncalcified aortic valve and small body habitus, after a thorough assessment, including meticulous annular measurements and appropriate valve
\end{abstract}


sizing, she underwent a transcatheter aortic valve replacement (TAVR) with complete resolution of both her $\mathrm{Al}$ and recurrent, severe $\mathrm{PH}$. This case highlights, in a single patient, reversal of "fixed" $\mathrm{PH}$ with adequate left ventricular unloading, that "moderate" Al by standard criteria is often "severe" and must be considered in a VAD patient with recurrent $\mathrm{PH}$, and the need for meticulous pre-procedural planning for TAVR in patients with VADs, including accurate measurements of the aortic annulus to ensure adequate oversizing of the valve.

Keywords: HVAD, LVAD, TAVR, mechanical circulatory support, pulmonary hypertension, aortic insufficiency

Currently, more than $40 \%$ of patients undergo bridging with a durable ventricular assist device (VAD) prior to heart transplantation for various reasons, including for the presence of severe, "fixed" pulmonary hypertension. ${ }^{1}$ However, a bridging strategy with a VAD has potential limitations. In the perioperative phase of the VAD implant, the need for blood product transfusions may result in human leukocyte antigen (HLA) antibody sensitization, thus potentially limiting the recipient donor pool. ${ }^{2}$ Moreover, VAD-related complications may ensue while on VAD support, ${ }^{3}$ which may impact transplant candidacy or create new obstacles to overcome to achieve a successful transplant outcome. Herein, we report on a challenging case that highlights each of these obstacles and the strategies employed to overcome them, all of which occurred in the same patient.

A 43-year-old woman (body surface area of $1.6 \mathrm{~m}^{2}$; body mass index of 24.3 $\mathrm{kg} / \mathrm{m}^{2}$ ) with a dilated cardiomyopathy underwent Heartware ${ }^{\circledR}$ ventricular assist device (HVAD; Heartware International, Inc., Framingham, MA) implant for endstage heart failure as a bridge to heart transplantation. A bridge strategy was employed because, in addition to having New York Heart Association Class IV heart failure symptoms, we diagnosed severe pulmonary hypertension $(\mathrm{PH})$ and found her to be highly sensitized, which would likely require a long wait time on the transplant list. With respect to her allosensitization status, she was highly sensitized with major histocompatibility complex (MHC) Class $\mathrm{I}=100 \%$ and Class $\|=100 \%$. Regarding her severe $\mathrm{PH}$, right heart catheterization $(\mathrm{RHC})$ prior to VAD implant revealed a pulmonary artery pressure (PAP) of $67 / 33 / 47 \mathrm{~mm} \mathrm{Hg}$, a pulmonary capillary wedge pressure (PCWP) of $26 \mathrm{~mm} \mathrm{Hg}$, a cardiac output (CO) by thermodilution of $2.12 \mathrm{~L} / \mathrm{min}$, and a pulmonary vascular resistance (PVR) of 9.9 Wood units, which did not significantly improve during acute vasodilator testing with intravenous nitroprusside. Pre-operative echocardiography revealed a normal sized aortic root with no aortic insufficiency (Al). She underwent a successful HVAD implant and her pre-discharge echocardiogram revealed an adequately unloaded left ventricle with a closed aortic valve throughout the cardiac cycle.

Approximately one year after HVAD implant, despite what has previously been coined "fixed" $\mathrm{PH}$ defined as the presence of a PVR $>5$ Woods units, a transpulmonary gradient $>15 \mathrm{~mm} \mathrm{Hg}$, and non-reversible hemodynamics with vasodilator administration, ${ }^{4}$ her $\mathrm{PH}$ had nearly resolved entirely. Specifically, her remeasured hemodynamics during a subsequent $\mathrm{RHC}$ demonstrated a PAP of $34 / 11 / 20 \mathrm{~mm} \mathrm{Hg}$ and a PCWP of $11 \mathrm{~mm} \mathrm{Hg}$. As the patient was not treated with any $\mathrm{PH}$-targeted medical therapies during this time span, the reversibility of her 
severe $\mathrm{PH}$, which some may have been inclined to consider to be "fixed" or irreversible, could be attributed to the impact of excellent left ventricular unloading of sufficient duration. Despite efforts at desensitization with plasmapheresis, IVIG, and bortezomib she remained with MHC Class I and Class II antigens of $100 \%$. However, many of her titers reduced sufficiently with the desensitization treatments to allow us to continue to consider her for heart transplantation, which she strongly desired.

Two years after VAD implantation, while continuing to wait for an acceptable HLA matched donor, she began to experience a gradual increase in dyspnea. Repeat $\mathrm{RHC}$ revealed a recurrence of her severe $\mathrm{PH}$ but also now with evidence of inadequate left ventricular unloading. Specifically, she had a PAP of 60/30/43 mm $\mathrm{Hg}$, a PCWP of $19 \mathrm{~mm} \mathrm{Hg}, \mathrm{CO}$ by thermodilution of $3.07 \mathrm{~L} / \mathrm{min}$ and a PVR of 7.8 Wood units. There was no evidence for VAD thrombosis, her blood pressure was well controlled, and increases in her VAD speed did not lower her PCWP (or PAPs) further as would normally be expected to occur. Echocardiography revealed the presence of new, at least "moderate" aortic insufficiency $(\mathrm{Al})$ by standard grading criteria (Figure 1$)^{5}$ in the setting of a normal sized aortic root and no overt aortic leaflet pathology. However, we were convinced that the continuous nature of the "moderate" Al in this clinical context was sufficient to explain her heart failure symptoms, elevated left sided filling pressures and recurrence of her severe $\mathrm{PH},{ }^{6}$ which rendered her ineligible yet again for proceeding with transplantation.

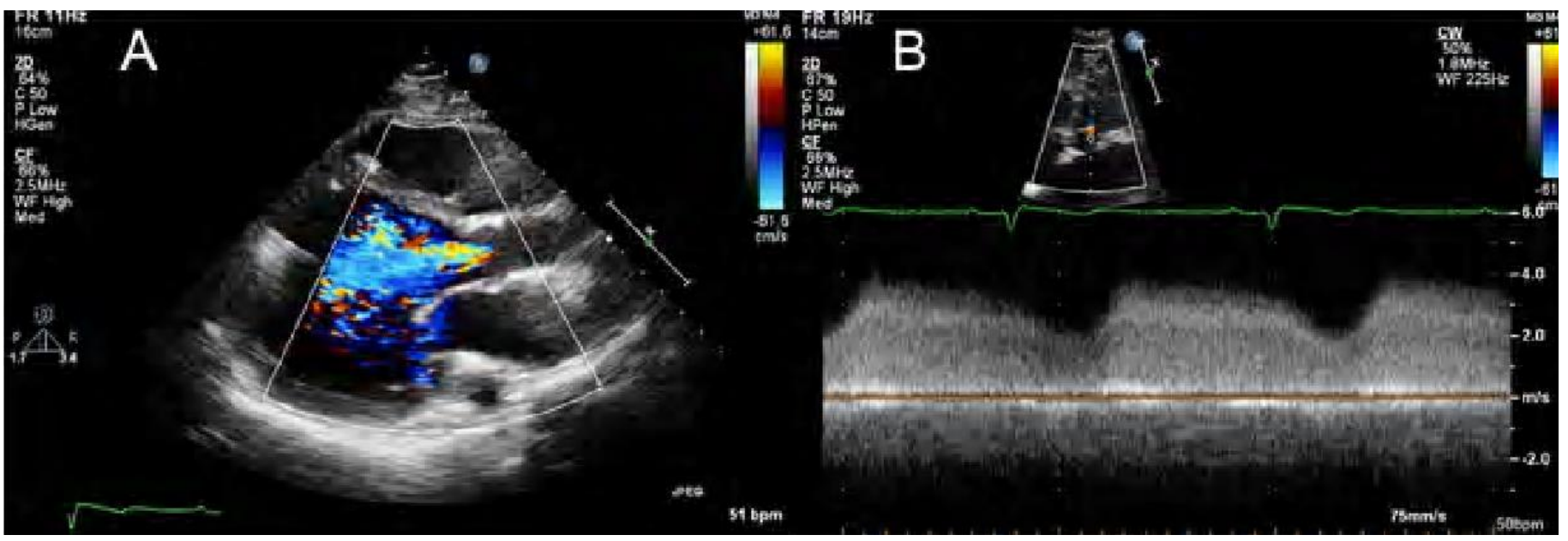

Figure 1. Evaluation of aortic insufficiency by echocardiogram. Panel A shows at least moderate aortic insufficiency by color Doppler in the parasternal long axis view. Panel B illustrates the continuous flow nature of the aortic insufficiency by interrogation with continuous wave Doppler.

We decided to pursue transcatheter aortic valve replacement (TAVR) as the least invasive means by which to treat her Al while limiting her risk of surgical complications including the possibility of requiring additional blood products. 
However, TAVR for AI, especially in a patient with a VAD and a non-ischemic cardiomyopathy, can be fraught with procedural complications, such as valve migration in the setting of a non-calcified aortic valve. Among the strategies to consider includes slightly oversizing the aortic valve which is critical to ensure sufficient anchoring to reduce the risk of migration while also optimizing valve function and hemodynamics. ${ }^{7}$ In this patient, careful pre-procedural planning to ensure safe valve placement included the temporary addition of intravenous dobutamine $5 \mathrm{u} / \mathrm{kg} / \mathrm{min}$ and the transient lowering of the patient's VAD speed from 2800 RPM to 2300 RPM, both of which were required to induce aortic valve opening in an effort to precisely measure her aortic annulus. Ultimately, a TAVR was performed via a right transfemoral approach with a Medtronic (Medtronic, Dublin, Ireland) Evolut ${ }^{\mathrm{TM}}$ Pro $26 \mathrm{~mm}$ valve. There were no procedural complications and the final aortogram revealed a well seated valve with no Al (Figure 2).

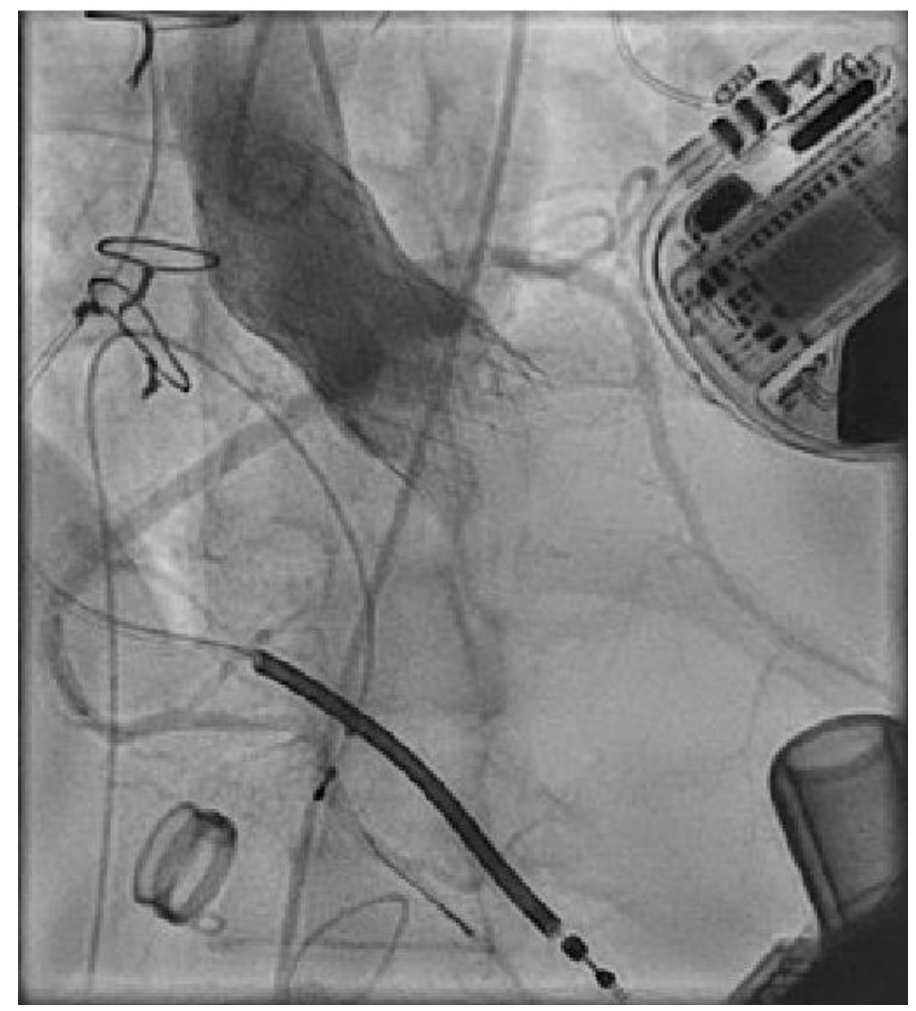

Figure 2. Aortography after transcatheter aortic valve replacement. In the Left Anterior Oblique 30 degrees and Cranial 15 degrees view, aortography shows a well-seated, bioprosthetic aortic valve without evidence of aortic insufficiency and without compression of the left or right coronary arteries.

At six weeks following her TAVR, her symptoms had resolved entirely. Repeat $\mathrm{RHC}$ revealed a normalization of her PAPs with a PAP of $31 / 10 / 18 \mathrm{~mm} \mathrm{Hg}$, a PCWP of $6 \mathrm{~mm} \mathrm{Hg}$, and a CO of $3.7 \mathrm{~L} / \mathrm{min}$. (Table 1 and Figure 3). Repeat echocardiography confirmed a well-seated valve and complete absence of Al. Her eligibility for transplant was subsequently re-opened. 
Table 1. Serial invasive hemodynamic measurements with right heart catheterization at critical time points in the patient's clinical course

$\begin{array}{lcccc}\text { Right Heart } & \text { Prior to } & \text { One year } & \text { Two years after } & \\ \text { Catheterization } & \text { LVAD } & \text { after LVAD } & \text { LVAD implant } & \text { Six weeks } \\ \text { implant } & \text { implant } & \text { with "moderate" } & \text { after TAVR }\end{array}$

\begin{tabular}{lcccc}
\hline $\begin{array}{l}\text { Pulmonary artery } \\
\text { pressure (mean), } \\
\text { mm Hg }\end{array}$ & $67 / 33(47)$ & $34 / 11(20)$ & $60 / 30(43)$ & $31 / 10(18)$ \\
$\begin{array}{l}\text { Pulmonary } \\
\text { capillary wedge } \\
\text { pressure, mm Hg }\end{array}$ & 26 & 11 & 19 & 6 \\
$\begin{array}{l}\text { Transpulmonary } \\
\text { pressure } \\
\text { gradient, mm Hg }\end{array}$ & 21 & 9 & 24 & 12 \\
$\begin{array}{l}\text { Cardiac output } \\
\text { by } \\
\text { thermodilution, } \\
\text { mm Hg }\end{array}$ & 2.1 & 3.4 & 3.1 & 3.7 \\
$\begin{array}{l}\text { Pulmonary } \\
\text { vascular } \\
\text { resistance, } \\
\text { Woods units }\end{array}$ & 9.9 & 2.6 & & 3.5 \\
& & & 7.8 &
\end{tabular}

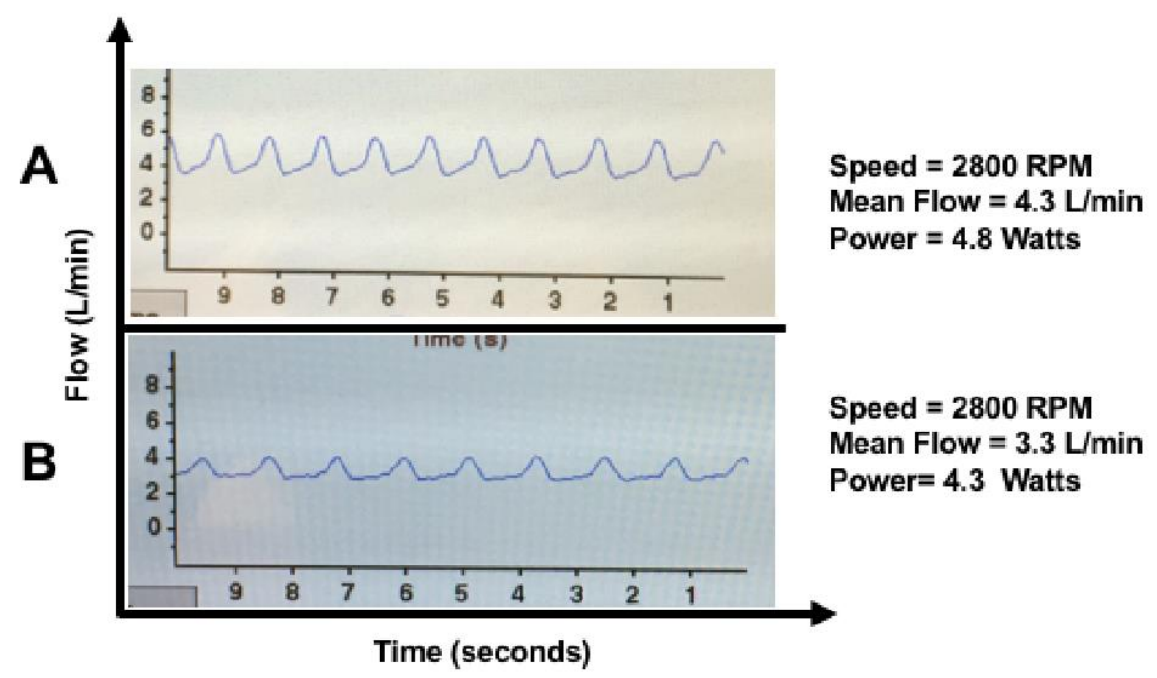

Figure 3. HVAD waveforms before and after transcatheter valve replacement. Immediately prior to transcatheter aortic valve replacement (Panel A), a modest but higher mean flow was present owed to the regurgitant aortic insufficiency volume which dropped immediately after transcatheter aortic valve replacement (Panel $B$ ) due to the elimination of the regurgitant flow through the pump. 
This case illustrates several key teaching points. First, $\mathrm{PH}$ in left heart failure is nearly always due to persistent elevations in left sided filling pressures yet is also nearly always reversible once the filling pressures are lowered, even in the presence of a severely elevated, "fixed" PVR. ${ }^{4}$ Indeed, our patient's severe PH was due to her left heart failure and reversed shortly following her VAD implant. When a recurrence of her severe $\mathrm{PH}$ occurred, particularly in the setting of elevated left sided pressures, a limited and finite list of culprit possibilities exist in a VAD patient, one of which is AI. By eliminating the AI via TAVR and consequently re-normalizing her left-sided filling pressures, her severe $\mathrm{PH}$ again resolved. Second, diagnosing de novo Al and its management in patients with continuous flow VADs deserves special attention. Clinically important AI in VAD supported patients may not meet standard severe Al grading criteria, thus requiring a high index of suspicion when present such as in our patient whose pre-TAVR imaging consistently suggested her Al to be "moderate" and not "severe". The inability to unload the LV despite increases in VAD speed and the presence of novel, echocardiographic parameters ${ }^{8}$ may be two potential indicators of its true severity. Third, the absence of aortic valve opening at time of discharge after LVAD implant has been associated with increased risk of the development of Al and may have contributed in this case.$^{9}$ However, the vast majority of patients with an LVAD will not develop clinically significant Al even in the absence of intermittent aortic valve opening. This patient required a sufficient VAD speed to maximally unload the LV to induce reversibility of her $\mathrm{PH}$; moreover, occasional attempts to lower her VAD speed resulted in a recurrence of heart failure symptoms, making the decision to maintain a higher VAD speed appropriate. Lastly, TAVR evaluation in the setting of a VAD requires careful pre-procedure planning regarding the valve type, valve size, and intra-procedure monitoring for valve migration. In particular, meticulous efforts aimed at precisely measuring aortic annulus dimensions and a slight oversizing of the aortic valve may be critical in VAD patients undergoing TAVR.

\section{References}

1. Chambers DC, Yusen RD, Cherikh WS, et al. The Registry of the International Society for Heart and Lung Transplantation: Thirty-fourth Adult Lung And Heart-Lung Transplantation Report-2017; Focus Theme: Allograft ischemic time. J Heart Lung Transplant. 2017;36(10):1047-1059.

2. Kobashigawa J, Mehra M, West L, et al. Report from a consensus conference on the sensitized patient awaiting heart transplantation. $J$ Heart Lung Transplant. 2009;28(3):213-225.

3. Cowger J, Pagani FD, Haft JW, Romano MA, Aaronson KD, Kolias TJ. The development of aortic insufficiency in left ventricular assist devicesupported patients. Circ Heart Fail. 2010;3(6):668-674.

4. Koulova A, Gass AL, Patibandla S, Gupta CA, Aronow WS, Lanier GM. Management of pulmonary hypertension from left heart disease in candidates for orthotopic heart transplantation. $J$ Thorac Dis. 2017;9(8):2640-2649. 
5. Zoghbi WA, Adams D, Bonow RO, et al. Recommendations for Noninvasive Evaluation of Native Valvular Regurgitation: A Report from the American Society of Echocardiography Developed in Collaboration with the Society for Cardiovascular Magnetic Resonance. J Am Soc Echocardiogr. 2017;30(4):303-371.

6. Basu B, Cherian G, Krishnaswami S, Sukumar IP, John S. Severe pulmonary hypertension in advanced aortic valve disease. Br Heart $\mathrm{J}$. 1978;40(11):1310-1313.

7. Rene AG, Desai N, Wald J, Rame JE, Frogel JK, Anwaruddin S. Transfemoral transcatheter aortic valve replacement with a self-expanding valve for severe aortic regurgitation in a patient with left ventricular assist device. J Card Surg. 2017;32(11):741-745.

8. Grinstein J, Kruse E, Sayer G, et al. Accurate Quantification Methods for Aortic Insufficiency Severity in Patients With LVAD: Role of Diastolic Flow Acceleration and Systolic-to-Diastolic Peak Velocity Ratio of Outflow Cannula. JACC Cardiovasc Imaging. 2016;9(6):641-651.

9. Jorde UP, Uriel N, Nahumi N, et al. Prevalence, significance, and management of aortic insufficiency in continuous flow left ventricular assist device recipients. Circ Heart Fail. 2014;7(2):310-319. 